\title{
Decentralized Stability Conditions for Inverter-Based Microgrids
}

\author{
Khaled Laib, Jeremy Watson and Ioannis Lestas
}

\begin{abstract}
We consider the problem of stability analysis of an inverter-based microgrid where higher order models are used for the inverter and line dynamics. Decentralized conditions are derived through which stability of the network can be deduced, with these formulated as input/output conditions on locally defined subsystems. The conditions derived allow to exploit the natural passivity properties of lines when these are represented in a common reference frame, but reduce the conservatism by additionally taking into account the coupling with neighbouring buses. Examples are given to demonstrate the results presented.
\end{abstract}

\section{INTRODUCTION}

There has been an increasing integration of renewable energy in recent years for environmental and economic reasons. The significance of microgrids in this context has been reported in the literature, with these expected to have the ability to operate autonomously, either due to a disconnection from the main grid or when providing support to remote communities [1], [2].

Renewable energy units and storage devices are usually interfaced to AC grids by means of DC/AC inverters and the corresponding microgrid is referred to as an inverter-based microgrid. In these microgrids, new challenges associated with modeling and control/analysis are encountered relative to more conventional grids, which rely primarily on rotating machines with high inertia. For instance, inverters and distribution lines have comparable response times and hence the usual timescale separation modeling approaches used in conventional grids are not always applicable [2], [3].

Existing inverter based microgrid studies include the analysis and simulation of advanced inverter models in specific configurations or the use of reduced order models for the line and inverter dynamics in order to establish network wide stability results [4], [5], [6], [7]. An important property in this context is also the fact that when AC grid dynamics are represented in a common reference frame the line dynamics preserve their natural passivity properties and therefore passivation of bus dynamics can ensure stability of the network [8], [9]. Nevertheless, the coupling between voltage and frequency dynamics in inverters and their faster timescales render inverters multivariable systems for which passivation can be often hard to achieve and hence alternative approaches need to be investigated.

In this paper we focus on the derivation of decentralized conditions through which asymptotic stability of an AC microgrid can be established. The analysis is carried out on models that maintain higher order dynamics for the

This work was supported by ERC starting grant 679774 .

The authors are with the Department of Engineering, University of Cambridge, UK. Emails: <k1507, jdw69, icl20>@cam.ac.uk inverters and the lines connecting them. In particular, we consider different decompositions of the microgrid that lead to decentralized conditions for stability, which are formulated as input/output conditions on locally defined subsystems. A main contribution is to show that these conditions can be combined using appropriate homotopy arguments thus reducing the conservatism in the analysis. In particular, the conditions derived allow to exploit the passivity properties of lines in a common reference frame, but reduce the conservatism by taking also into account the strength of the coupling with neighbouring buses.

Outline: The paper is structured as follows. Section II presents the inverter-based microgrid model and the problem setting. Section III presents the main results of the paper followed by examples in Section IV. Conclusions are drawn in Section V.

Notation: The set of real and complex numbers are denoted $\mathbb{R}$ and $\mathbb{C}$ respectively. The set of real positive numbers including 0 and $+\infty$ is denoted $\overline{\mathbb{R}}^{+}=\mathbb{R}^{+} \cup\{+\infty\}$ where $\mathbb{R}^{+}$is the set of real positive numbers. The closed right half plane including the imaginary axis and infinity is denoted by $\overline{\mathbb{C}}^{+}$. For a real/complex matrix $M \in \mathbb{R}^{n \times n} / M \in$ $\mathbb{C}^{n \times n}$, its transpose and its conjugate are denoted by $M^{T}$ and $M^{*}$ respectively. The identity matrix is denoted $I$ and its dimension are deduced form the context. The set of stable rational transfer function matrices is denoted $\mathbb{R H}_{\infty}$ with its dimensions similarly deduced from the context. The Kronecker product of two matrices $M_{i}$ and $M_{j}$ is denoted by $M_{i} \otimes M_{j}$. The direct sum of matrices $M_{i}$ with $i=1, \ldots, n$ is denoted by $\oplus_{i=1}^{n} M_{i}$. Finally, for complex matrices $X, \Pi_{11}, \Pi_{12}, \Pi_{22}$ with compatible dimensions and $\epsilon \geq 0$, we use $X \in \mathbf{Q C}(\Pi, \epsilon)$ to denote

$$
\left(\begin{array}{c}
X \\
I
\end{array}\right)^{*} \underbrace{\left(\begin{array}{ll}
\Pi_{11} & \Pi_{12} \\
\Pi_{12}^{*} & \Pi_{22}
\end{array}\right)}_{\Pi}\left(\begin{array}{c}
X \\
I
\end{array}\right) \geq \epsilon I
$$

and $X \in \overline{\mathbf{Q C}}(\Pi, \epsilon)$ to denote

$$
\left(\begin{array}{c}
I \\
-X
\end{array}\right)^{*}\left(\begin{array}{ll}
\Pi_{11} & \Pi_{12} \\
\Pi_{12}^{*} & \Pi_{22}
\end{array}\right)\left(\begin{array}{c}
I \\
-X
\end{array}\right) \leq-\epsilon I
$$

\section{MODELS AND PROBLEM SETTINGS}

\section{A. Preliminaries}

The notation $z^{a b c}(t): \mathbb{R} \rightarrow \mathbb{R}^{3}$ is used to represent threephase AC signals given by

$$
z^{a b c}(t)=\sqrt{2}|z(t)|\left(\begin{array}{c}
\cos (\theta(t)) \\
\cos \left(\theta(t)-\frac{2}{3} \pi\right) \\
\cos \left(\theta(t)+\frac{2}{3} \pi\right)
\end{array}\right)
$$

with $|z(t)|$ as the amplitude and $\theta(t)$ as the angle. Note that the different three-phase AC signals $z^{a b c}(t)$ considered in 
this paper are voltages and currents. The Park transformation $P(\theta(t))$ is used to map three-phase AC signal components into their $0 d q$ coordinates. The resulting signal is denoted $z^{0 d q}(t)$ and is given by $z^{0 d q}(t)=\sqrt{\frac{2}{3}} P(\theta(t)) z^{a b c}(t)$ with $P(\theta(t))=\left(\begin{array}{ccc}\frac{1}{\sqrt{2}} & \frac{1}{\sqrt{2}} & \frac{1}{\sqrt{2}} \\ \cos (\theta(t)) & \cos \left(\theta(t)-\frac{2 \pi}{3}\right) & \cos \left(\theta(t)+\frac{2 \pi}{3}\right) \\ \sin (\theta(t)) & \sin \left(\theta(t)-\frac{2 \pi}{3}\right) & \sin \left(\theta(t)+\frac{2 \pi}{3}\right)\end{array}\right)$

Note that the matrix $P(\theta(t))$ is orthogonal, that is $P(\theta(t))^{-1}=P(\theta(t))^{T}$.

Assumption 1 The microgrid AC signals are symmetric and balanced three-phase AC signals.

Assumption 1 results in $z^{a}(t)+z^{b}(t)+z^{c}(t)=0$ and therefore $z^{0 d q}(t)$ is reduced to its $d q$ components (second, third components of the vector) denoted by $z^{d q}(t)$. If for a three phase signal at a bus $j$ the angle $\theta(t)$ in (2) is the angle of the voltage at that bus then, $z^{d q}(t)$ is referred to as a representation in the local reference frame. If in (2) the angle $\theta(t)$ is replaced by an angle $\theta_{c}(t)$ that satisfies $\dot{\theta}_{c}(t)=\omega_{c}$ where $\omega_{c}$ is a constant (the synchronous frequency), then the corresponding transform $z^{0 D Q}(t)=\sqrt{\frac{2}{3}} P\left(\theta_{c}(t)\right) z^{a b c}(t)$ is referred to as representation in the common reference frame. The link between the two transforms is given by $z_{j}^{d q}(t)=$ $T\left(\delta_{j}(t)\right) z_{j}^{D Q}(t)$ and $z_{j}^{D Q}(t)=T\left(\delta_{j}(t)\right)^{-1} z_{j}^{d q}(t)$ where

$$
T\left(\delta_{j}(t)\right)=\left(\begin{array}{cc}
\cos \left(\delta_{j}(t)\right) & \sin \left(\delta_{j}(t)\right) \\
-\sin \left(\delta_{j}(t)\right) & \cos \left(\delta_{j}(t)\right)
\end{array}\right)
$$

with $\delta_{j}(t)=\theta_{j}(t)-\theta_{c}(t)$ with $\theta_{j}(t)$ the voltage angle at bus $j$. Note that the matrix $T\left(\delta_{j}(t)\right)$ is also orthogonal, that is $T\left(\delta_{j}(t)\right)^{-1}=T\left(\delta_{j}(t)\right)^{T}$.

The inverter-based microgrid is a power system comprised of $n_{b}$ buses and $n_{\ell}$ distribution lines, which we represent as a connected graph $(\mathcal{N}, \mathcal{E})$ where $\mathcal{N}=\left\{1,2, \ldots, n_{b}\right\}$ is the set of buses and $\mathcal{E}=\left\{1, \ldots, n_{\ell}\right\} \subset \mathcal{N} \times \mathcal{N}$ is the set of edges (lines). A direction is assigned to each edge which can be arbitrarily chosen. The corresponding $n_{b} \times n_{\ell}$ incidence matrix is denoted $\mathcal{A}$ and it is given by

$$
\mathcal{A}_{j k}=\left\{\begin{array}{cl}
-1 & \text { if edge } k \text { leaves bus } j \\
+1 & \text { if edge } k \text { enters bus } j \\
0 & \text { otherwise. }
\end{array}\right.
$$

Each bus includes a DC/AC inverter with its controller and we assume that the loads are connected to the inverter terminals. The current through the $k^{t h}$ line, is represented in the common reference frame and is denoted by $i_{\ell_{k}}^{D Q}(t)$. In particular, this denotes the current with the same direction as that of the edge $k$. The input current at the $j^{\text {th }}$ bus, represented in the local reference frame, is denoted by $i_{j}^{d q}(t)$ and in the common reference frame by $i_{j}^{D Q}(t)$. Similarly, its output voltage in the two reference frames is denoted by $v_{j}^{d q}(t)$ and $v_{j}^{D Q}(t)$ respectively. The voltage vector of all buses is denoted $V_{B}^{D Q}(t)=\left(v_{j}^{D Q}(t)\right)_{j \in \mathcal{N}}$ and the vector of all line currents is denoted $I_{L}^{D Q}(t)=\left(i_{\ell_{k}}^{D Q}(t)\right)_{k \in \mathcal{E}}$.

\section{B. Line dynamics}

In this paper distribution lines are modelled as RL components. The dynamics of the current $i_{\ell_{k}}^{D Q}(t)$ through the line $\ell_{k}$, with $k \in \mathcal{E}$, are given by

$$
\frac{\mathrm{d} i_{\ell_{k}}^{D Q}(t)}{\mathrm{d} t}=\left(\begin{array}{cc}
\frac{-r_{k}}{l_{k}} & \omega_{c} \\
-\omega_{c} & \frac{-r_{k}}{l_{k}}
\end{array}\right) i_{\ell_{k}}^{D Q}(t)+\frac{1}{l_{k}} \delta V_{\ell_{k}}^{D Q}(t)
$$

where $r_{k}$ and $l_{k}$ are the $k^{t h}$ line resistance and inductance, $\omega_{c}$ is the constant synchronous frequency and $\delta V_{\ell_{k}}^{D Q}(t)$ is the voltage difference between line $\ell_{k}$ upstream and downstream.

\section{Bus dynamics}

We present now a general bus model to account for a broad class of DC/AC inverters, controllers and loads. These dynamics are allowed to be different at each bus $j$. DC/AC inverters are composed of three main stages: DC stage, switching stage and AC stage. Some assumptions are usually encountered in the inverter-based microgrid literature to derive simple models and controllers.

Assumption 2 The DC stage is equipped with sufficient energy reserves and the switching is performed at a frequency much higher than the natural frequencies of the inverter. Furthermore, the power generated on the DC stage is transferred to the AC stage without switching losses.

Under Assumption 2, it is possible to use an averaged inverter model in order to design controllers satisfying some requirements: output voltage regulation, power sharing, etc.. Several controllers for inverter-based power systems have been proposed in the literature using different techniques such as: virtual synchronous generators, angle droop, matching control, etc., see [3].

We consider that each inverter is already equipped with its controller and is connected to its load. The dynamics of the $j^{t h}$ bus can thus be described as a two input two output dynamical system with $i_{j}^{d q}(t)$ as inputs and $v_{j}^{d q}(t)$ as outputs. These dynamics are given as follows

$$
\begin{aligned}
\frac{\mathrm{d} x_{j}^{d q}(t)}{\mathrm{d} t} & =f_{j}\left(x_{j}^{d q}(t), i_{j}^{d q}(t)\right) \\
v_{j}^{d q}(t) & =g_{j}\left(x_{j}^{d q}(t), i_{j}^{d q}(t)\right)
\end{aligned}
$$

where $x_{j}^{d q}(t) \in \mathbb{R}^{2 n_{x_{j}}}$ is the state space variable vector considered at each bus (inverter variables, controller variables and load variables) and $f_{j}$ and $g_{j}$ are vector functions such that $f_{j}: \mathbb{R}^{2 n_{x_{j}}} \times \mathbb{R}^{2} \rightarrow \mathbb{R}^{2 n_{x_{j}}}$ and $g_{j}: \mathbb{R}^{2 n_{x_{j}}} \times \mathbb{R}^{2} \rightarrow \mathbb{R}^{2}$.

In order to derive stability results for the microgrid, both the line and the bus dynamics have to be expressed in the same reference frame, which is chosen here as the common reference frame. Therefore and after expressing the $j^{\text {th }}$ bus input-output signals $i_{j}^{d q}(t)$ and $v_{j}^{d q}(t)$ in the common reference frame, the $j^{\text {th }}$ bus model becomes

$$
\begin{aligned}
& \frac{\mathrm{d}}{\mathrm{d} t}\left(\begin{array}{c}
\delta_{j}(t) \\
x_{j}^{d q}(t)
\end{array}\right)=\left(\begin{array}{c}
h_{j}\left(x_{j}^{d q}(t), T\left(\delta_{j}(t)\right) i_{j}^{D Q}(t)\right) \\
f_{j}\left(x_{j}^{d q}(t), T\left(\delta_{j}(t)\right) i_{j}^{D Q}(t)\right)
\end{array}\right) \\
& v_{j}^{D Q}(t)=T\left(\delta_{j}(t)\right)^{-1} g_{j}\left(x_{j}^{d q}(t), T\left(\delta_{j}(t)\right) i_{j}^{D Q}(t)\right) \\
& \text { where } h_{j}: \mathbb{R}^{2 n_{x_{j}}} \times \mathbb{R}^{2} \rightarrow \mathbb{R} .
\end{aligned}
$$




\section{Power system small signal model}

As it can be seen from (4), the bus model is nonlinear which makes the microgrid model nonlinear. Equilibirum points can be found by setting the time derivatives in (3)(4) to zero then solve the resulting system of equations. Thereafter, a linearisation can be performed using an obtained equilibrium. For this purpose, we require a smoothness assumption to enable linearisation to be taken.

Assumption 3 The function $h_{j}$ and the vector functions $f_{j}$ and $g_{j}$ in (4) are locally Lipschitz around the considered equilibrium of (3)-(4).

Under Assumption 3, the system (3)-(4) can be linearized about the equilibrium being considered. In order to analyse this linearisation, let $\bar{q}(t)=q(t)-\left.q\right|_{e q}$ denote the deviation of any quantity $q(t)$ from its value $\left.q\right|_{e q}$ at this equilibrium.

We assume that the dynamics (4) when linearized are stabilizable and detectable. Finally, we adopt an input/output representation of the small signal model of inverter-based microgrid (3)-(4), and we introduce the following two sets of transfer function matrices.

- $L_{k}(s)$ is the transfer function matrix of the line dynamics (3) from the inputs $\overline{\delta V_{\ell_{k}}^{D Q}}(t)$ to the outputs $\overline{i_{\ell_{k}}^{D Q}}(t)$

- $B_{j}(s)$ is the transfer function matrix for the linearized versions of the bus dynamics (4) from the inputs $\overline{i_{j}^{D Q}}(t)$ to the outputs $\overline{v_{j}^{D Q}}(t)$.

Note that the different $L_{k}(s)$ are in $\mathbb{R H}_{\infty}^{2 \times 2}$ as the matrix $\left(\begin{array}{cc}-r_{k} / l_{k} & \omega_{c} \\ -\omega_{c} & -r_{k} / l_{k}\end{array}\right)$ in (3) is Hurwitz. We also assume that the individual bus dynamics $B_{j}(s)$ are asymptotically stable.

Assumption 4 For each bus $j, B_{j}(s) \in \mathbb{R H}_{\infty}^{2 \times 2}$.

Recalling that $\overline{\delta V_{\ell_{k}}^{D Q}}(t)=-\left(\left(a_{k}^{c}\right)^{T} \otimes I_{2}\right) \overline{V^{D Q}}(t)$ and $\overline{i_{j}^{D Q}}(t)=\left(a_{j}^{r} \otimes I_{2}\right) \overline{I_{L}^{D Q}}(t)$ where $a_{k}^{c}$ and $a_{j}^{r}$ are the $k^{t h}$ column and the $j^{\text {th }}$ row of the incidence matrix $\mathcal{A}$ respectively, then the small signal model of inverter-based microgrid (3)-(4) can be represented as a negative feedback interconnectiongiven by

$$
\begin{array}{ll}
\overline{I_{L}^{D Q}}(s)=-L(s)\left(\mathcal{A} \otimes I_{2}\right)^{T} & \overline{V_{B}^{D Q}}(s) \\
\overline{V_{B}^{D Q}}(s)=B(s)\left(\mathcal{A} \otimes I_{2}\right) & \overline{I_{L}^{D Q}}(s)
\end{array}
$$

with $B(s)=\oplus_{j=1}^{n_{b}} B_{j}(s)$ and $L(s)=\oplus_{k=1}^{n_{\ell}} L_{k}(s)$ where $B_{j}(s) \in \mathbb{R H}_{\infty}^{2 \times 2}$ and $L_{k}(s) \in \mathbb{R H}_{\infty}^{2 \times 2}$.

\section{MAIN RESULTS}

The following theorem gives sufficient conditions for the local asymptotic stability of the equilibrium $\left.q\right|_{e q}$ of the inverter-based microgrid (3)-(4) based on input/ouptut conditions on the subsystems in (5).

Theorem 1 Suppose that Assumptions 1-4 are all satisfied and consider the inverter-based microgrid system (3)-(4) with its small signal model around a considered equilibrium $\left.q\right|_{e q}=\left(\left(\left.I_{L}^{D Q}\right|_{e q}\right)^{T},\left(\left.V_{B}^{D Q}\right|_{e q}\right)^{T},\left(\left(\left.\delta_{j}\right|_{e q}\right)_{j \in N}\right)^{T},\left(\left(\left.x_{j}^{D Q}\right|_{e q}\right)_{j \in N}\right)^{T}\right)$ represented as in (5).
The equilibrium $\left.q\right|_{e q}$ is locally asymptotically stable if for each $\omega \in \overline{\mathbb{R}}^{+}$, at least one of the following two statements is satisfied.

- Statement 1: There exist scalars $\epsilon_{G_{j}}(\omega)>0, j=$ $1 \ldots n_{b}$ and $2 \times 2$ complex matrices $\Pi_{11}^{k}(\mathbf{j} \omega) \leq 0, \Pi_{12}^{k}(\mathbf{j} \omega)$ and $\Pi_{22}^{k}(\mathbf{j} \omega) \geq \epsilon_{G_{j}}(\omega) I_{2 n_{b}}, \quad k=1, \ldots, n_{\ell}$ satisfying $-\Pi_{12}^{k}(\mathbf{j} \omega)-\Pi_{12}^{k}(\mathbf{j} \omega)^{*}+2 \Pi_{22}^{k}(\mathbf{j} \omega) \leq 0$, such that

$$
G_{j}(\mathbf{j} \omega) \in \mathbf{Q C}\left(\Pi(\mathbf{j} \omega), \epsilon_{G_{j}}(\omega)\right) \quad j \in \mathcal{N}
$$

with $G_{j}(\mathbf{j} \omega)=L(\mathbf{j} \omega)\left(a_{j}^{r} \otimes I_{2}\right)^{T} B_{j}(\mathbf{j} \omega)\left(a_{j}^{r} \otimes I_{2}\right)$ and $\Pi(\mathbf{j} \omega)=\left(\begin{array}{cc}\oplus_{k=1}^{n_{l}} \Pi_{11}^{k}(\mathbf{j} \omega) & \oplus_{k=1}^{n_{l}} \Pi_{12}^{k}(\mathbf{j} \omega) \\ \oplus_{k=1}^{n_{l}} \Pi_{12}^{k}(\mathbf{j} \omega)^{*} & \oplus_{k=1}^{n_{l}=1} \Pi_{22}^{k}(\mathbf{j} \omega)\end{array}\right)$.

- Statement 2: There exist scalars $\epsilon_{B_{j}}(\omega)>0, j=1 \ldots n_{b}$ such that

$$
B_{j}(\mathbf{j} \omega) \in \mathbf{Q C}\left(\Pi^{P}, \epsilon_{B_{j}}(\omega)\right) \quad j \in \mathcal{N}
$$

with $\Pi^{P}=\left(\begin{array}{cc}0 & I_{2} \\ I_{2} & 0\end{array}\right)$.

The proof is provided in the appendix.

Remark 1 Both Statement 1 and Statement 2 are decentralized conditions that depend on local bus/line dynamics ${ }^{1}$. They are obtained using two different decomposition of the microgrid that lead to appropriate graph separation arguments [10]. In particular, Statement 2 is a more conventional passivity condition that involves individual bus dynamics. (e.g. [8]). Statement 1, is based on a different decomposition of the microgrid [10] that leads to subsystems involving each bus and the lines connected to it. A main contribution of Theorem 1 is to show that conditions (6) and (7) can be combined together pointwise over frequency by employing an appropriate homotopy argument exploited in the derivation, thus reducing the conservatism in the design.

Remark 2 Note that when considered individually, neither of the two conditions (6) and (7) is consistently less conservative compared to the other. For example, for any passive system $B_{j}$ Statement 2 holds for an arbitrarily large gain in contrast to Statement 1. On the other hand, Statement 2 does not take into account the 'strength' of the coupling among the bus dynamics at each frequency. Hence each condition has its own merits and Theorem 1 allows both conditions to be used simultaneously. Specific examples that demonstrate the relative merits of the two conditions will be discussed in the next section.

Remark 3 A design approach is to choose the two different conditions (6) and (7) at different frequency ranges, e.g. Statement 2 in regimes of higher gains, and Statement 1, with a choice of multipliers $\Pi_{i j}^{k}$, in regimes of weaker coupling and potential phase lags. The generalized KYP Lemma [11] can then be used for verification. It should be noted that this design specification can be seen as providing a grid code

\footnotetext{
${ }^{1}$ Note that due to the sparsity structure of $a_{j}^{r}$ for each bus $j, G_{j}$ involves only the neighbouring line dynamics $L_{k}$. Similarly multipliers $\Pi_{i j}^{k}$ are associated with each line $\ell_{k}$ and only the multipliers of neighbouring lines are relevant in (6) for each bus $j$.
} 
for the microgrid that needs to be decided a priori, i.e. local design rules at each bus which if satisfied ensures stability of a general network.

\section{EXAMPLES}

To demonstrate the efficiency of our results, we consider a two inverter-based microgrid where each bus is composed of a controlled DC/AC inverter connected to a load.

For graphical illustration purposes, we denote $\gamma_{j}(\omega)$ and $\nu_{j}(\omega)$ as the smallest eigenvalues of the expanded forms of conditions (6) and (7) respectively (i.e. left hand side of (1)), for $j \in\{1,2\}$. Within this context, Theorem 1 ensures stability if for all $\omega \in \overline{\mathbb{R}}^{+}$we have $\gamma_{j}(\omega)>0$ or $\nu_{j}(\omega)>0$.

The line dynamics are given by (3) where the incidence matrix is given by $\mathcal{A}=\left(\begin{array}{ll}-1 & 1\end{array}\right)^{T}$. The model, the control strategy and the numerical values in this example are taken from [9] (with these adapted to our context and notations).

The AC stage of each inverter is an LCL filter and its dynamics in its local $d q$ frame are given by (8) where $R_{f_{j}}$, $L_{f_{j}}, R_{c_{j}}, R_{c_{j}}, C_{f_{j}}, G_{f_{j}}$ and $\alpha_{j}^{f}$ are the filter parameters. $v_{f_{j}}^{d q}(t), i_{f_{j}}^{d q}(t), v_{c_{j}}^{d q}(t)$ and $i_{c_{j}}^{d q}(t)$ are internal filter voltages and current while $i_{j}^{d q}(t)$ is the current injection from the network lines and $v_{j}^{d q}(t)$ is the output voltage (see (9) bellow for its expression). $P_{j}(t)$ and $Q_{j}(t)$ are the active and reactive power respectively. $\eta_{j}(t)$ and $v_{K_{j}}^{d q}(t)$ are the time derivative of the angle $\theta_{j}(t)$ and the output of the filter controller respectively. Both will be specified later in each example depending on the adopted control strategy.

$$
\begin{aligned}
\frac{\mathrm{d} i_{f_{j}}^{d q}(t)}{\mathrm{d} t} & =\left(\begin{array}{cc}
\frac{-R_{f_{j}}}{L_{f_{j}}} & \eta_{j}(t) \\
-\eta_{j}(t) & \frac{-R_{f_{j}}}{L_{f_{j}}}
\end{array}\right) i_{f_{j}}^{d q}(t)+L_{f_{j}}^{-1}\left(v_{K_{j}}^{d q}(t)-v_{f_{j}}^{d q}(t)\right) \\
\frac{\mathrm{d} i_{c_{j}}^{d q}(t)}{\mathrm{d} t} & =\left(\begin{array}{cc}
\frac{-R_{c_{j}}}{L_{c_{j}}} & \eta_{j}(t) \\
-\eta_{j}(t) & \frac{-R_{c_{j}}}{L_{c_{j}}}
\end{array}\right) i_{c_{j}}^{d q}(t)+L_{f_{j}}^{-1}\left(v_{f_{j}}^{d q}(t)-v_{j}^{d q}(t)\right) \\
\frac{\mathrm{d} v_{c_{j}}^{d q}(t)}{\mathrm{d} t} & =\left(\begin{array}{cc}
\frac{-G_{f_{j}}}{C_{f_{j}}} & \eta_{j}(t) \\
-\eta_{j}(t) & \frac{-G_{f_{j}}}{C_{f_{j}}}
\end{array}\right) v_{f_{j}}^{d q}(t)+L_{f_{j}}^{-1}\left(i_{f_{j}}^{d q}(t)-i_{c_{j}}^{d q}(t)\right) \\
\frac{\mathrm{d} P_{j}(t)}{\mathrm{d} t} & =\alpha_{j}^{f}\left(-P_{j}(t)+v_{j}^{d}(t) i_{j}^{d}(t)+v_{j}^{q}(t) i_{j}^{q}(t)\right) \\
\mathrm{d} t & =\alpha_{j}^{f}\left(-Q_{j}(t)+v_{j}^{q}(t) i_{j}^{d}(t)-v_{j}^{d}(t) i_{j}^{q}(t)\right)
\end{aligned}
$$

The inverter is connected to a resistive load $R_{j}$ such that

$$
v_{j}^{d q}(t)=R_{j}\left(i_{j}^{d q}(t)+i_{c_{j}}^{d q}(t)\right)
$$

Finally, the angle $\delta_{j}(t)=\theta_{j}(t)-\theta_{c}(t)$ is used to express the input $i_{j}^{d q}(t)$ and the output $v_{j}^{d q}(t)$ in the common reference frame which leads to the additional dynamics

$$
\begin{aligned}
\frac{\mathrm{d} \delta_{j}(t)}{\mathrm{d} t} & =h_{j}\left(x_{j}^{d q}(t), i_{j}^{d q}(t)\right) \\
i_{j}^{d q}(t) & =\left(\begin{array}{cc}
\cos \left(\delta_{j}(t)\right) & \sin \left(\delta_{j}(t)\right) \\
-\sin \left(\delta_{j}(t)\right) & \cos \left(\delta_{j}(t)\right)
\end{array}\right) i_{j}^{D Q}(t) \\
v_{j}^{D Q}(t) & =\left(\begin{array}{cc}
\cos \left(\delta_{j}(t)\right) & -\sin \left(\delta_{j}(t)\right) \\
\sin \left(\delta_{j}(t)\right) & \cos \left(\delta_{j}(t)\right)
\end{array}\right) v_{j}^{d q}(t)
\end{aligned}
$$

where $h_{j}$ depends on the control strategy and it will be specified later in each example.

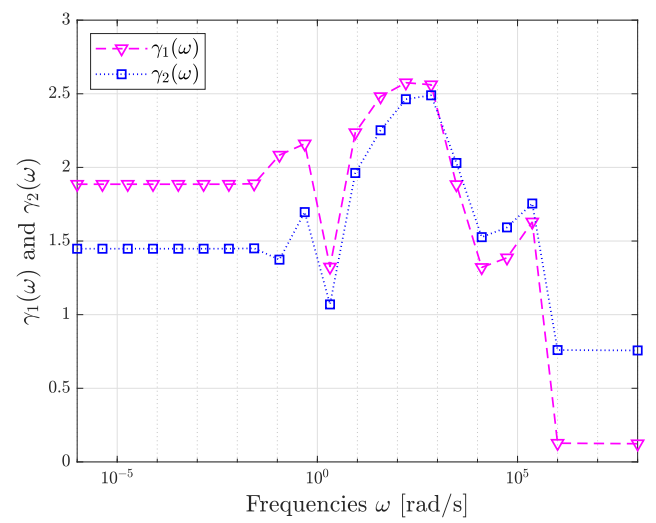

Fig. 1: Microgrid stability assessment using Statement 1.

\section{A. First controller: angle and voltage droop}

In this example, the angle droop technique is used to ensure synchronisation and active power sharing using the common reference frame angle (obtained via wireless communication), an active power constant $P_{c}$ and a control gain $m_{j}$ such that $\theta_{j}(t)=\theta_{c}(t)-m_{j}\left(P_{j}(t)-P_{c}\right)$. Therefore, $\eta_{j}(t)$ in model (8) is given by $\eta_{j}(t)=\omega_{c}+m_{j} \alpha_{j}^{f}\left(P_{j}(t)-\right.$ $\left.v_{j}^{d}(t) i_{j}^{d}(t)-v_{j}^{q}(t) i_{j}^{q}(t)\right)$ while $\delta_{j}(t)$ and $h_{j}\left(x_{j}^{d q}(t), i_{j}^{d q}(t)\right)$ used in (10) are given by $\delta_{j}(t)=-m_{j}\left(P_{j}(t)-P_{c}\right)$ and $h_{j}\left(x_{j}^{d q}(t), i_{j}^{d q}(t)\right)=m_{j} \alpha_{j}^{f}\left(P_{j}(t)-v_{j}^{d}(t) i_{j}^{d}(t)-v_{j}^{q}(t) i_{j}^{q}(t)\right)$.

The voltage droop technique is used to ensure a desired output voltage tracking. Therefore, the control input $v_{K_{j}}^{d q}(t)$ in model (8) is given as the output of the following controller

$$
\begin{aligned}
\frac{\mathrm{d} \zeta_{j}(t)}{\mathrm{d} t} & =v_{r}^{d q}(t)-v_{j}^{d q}(t) \\
\frac{\mathrm{d} \xi_{j}(t)}{\mathrm{d} t} & =a_{j}^{P}\left(v_{r}^{d q}(t)-v_{j}^{d q}(t)\right)+a_{j}^{I} \zeta_{j}(t)-i_{j}^{d q}(t) \\
v_{K_{j}}^{d q}(t) & =b_{j}^{P}\left(a_{j}^{P}\left(v_{r}^{d q}(t)-v_{j}^{d q}(t)\right)+a_{j}^{I} \zeta_{j}(t)-i_{j}^{d q}(t)\right)+b_{j}^{I} \xi_{j}(t)
\end{aligned}
$$

where $a_{j}^{P}, a_{j}^{I}, b_{j}^{P}$ and $b_{j}^{I}$ are control gains and $v_{r}^{d q}(t)$ is a reference to track.

Using Statement 1 in Theorem 1 and choosing the different $\Pi_{11}^{k}(\mathbf{j} \omega), \Pi_{12}^{k}(\mathbf{j} \omega)$ and $\Pi_{22}^{k}(\mathbf{j} \omega)$ to be scalars multiplied by $I_{2}$, it is possible to certify the stability of the microgrid as all $\gamma_{j}(\omega)>0$ for all $\omega \in \overline{\mathbb{R}}^{+}$as illustrated in Fig 1 .

Note that it was not possible to prove stability using the passivity argument of Statement 2 as the buses are not input strictly passive for all $\omega \in \overline{\mathbb{R}}^{+}$,

\section{B. Second controller: virtual impedance and state feedback}

In this second example, a virtual impedance technique is used to ensure power sharing by adjusting the voltage reference $v_{r}^{d q}(t)$ according to the input current $i_{j}^{d q}(t)$ such that $v_{r}^{d q}(t)=v_{N}^{d q}-Z_{j} i_{j}^{d q}(t)$ where $v_{N}^{d q}$ is a nominal voltage and $Z_{j}$ is a two by two constant matrix corresponding to a virtual impedance.

As discussed in [12], such a virtual impedance strategy is equivalent to angle droop, adjusting the output voltage angle based on active power and the voltage magnitude based 


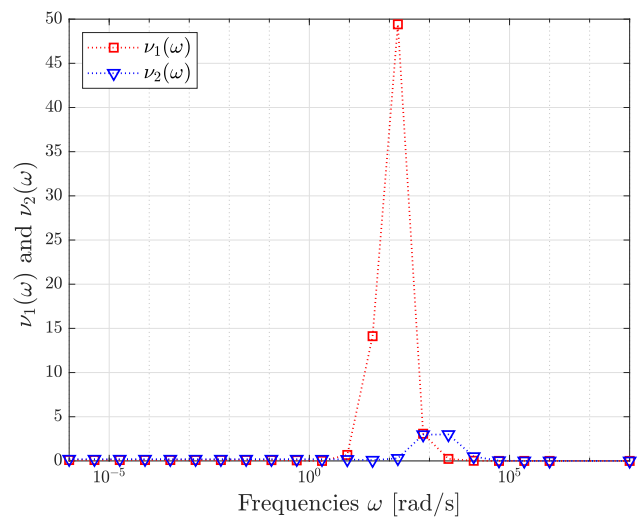

Fig. 2: Microgrid stability assessment using Statement 2.

on reactive power. Synchronization of inverters is ensured via some wireless communication by setting all the inverter frame angles to $\theta_{c}(t)$. Therefore, $\eta_{j}(t)$ in (8) is given by $\eta_{j}(t)=\omega_{c}$ while $\delta_{j}(t)$ and $h_{j}\left(x_{j}^{d q}(t), i_{j}^{d q}(t)\right)$ used in (10) are given by $\delta_{j}(t)=0$ and $h_{j}\left(x_{j}^{d q}(t), i_{j}^{d q}(t)\right)=0$.

Instead of the hierarchical proportional-integral loops used in traditional angle droop control, a state feedback controller is implemented to ensure a desired output voltage tracking and to obtain a suitable passivity property. The control input $v_{K_{j}}^{d q}(t)$ in (8) is given as the output of the following controller

$$
\begin{array}{ccc}
\frac{\mathrm{d} \zeta_{j}(t)}{\mathrm{d} t} & = & v_{r}^{d q}(t)-v_{j}^{d q}(t) \\
v_{K_{j}}^{d q}(t) & = & a_{j} i_{f_{j}}^{d q}(t)+b_{j} v_{r}^{d q}(t)+c_{j} i_{r}^{d q}(t)+d_{j} \zeta_{j}(t)
\end{array}
$$

where $a_{j}, b_{j}, c_{j}$, and $d_{j}$ are two by two control gains. More details on this control scheme and an evaluation of its performance will be included in future work.

It was possible to find such a control design such that the passivity based condition in Statement 2 of Theorem 1 holds and thus stability can be deduced. This is illustrated in Fig 2 where all $\nu_{j}(\omega)>0$ for all $\omega \in \overline{\mathbb{R}}^{+}$.

On the other hand, for this control design it was not possible to find $\Pi_{11}^{k}(\mathbf{j} \omega), \Pi_{12}^{k}(\mathbf{j} \omega)$ and $\Pi_{22}^{k}(\mathbf{j} \omega)$ that are scalars multiplied by $I_{2}$ (as in the first example) such that Statement 2 holds for all frequencies, due to the relatively large gains involved.

\section{CONCLUSION}

We have derived decentralized stability conditions for an inverter based microgrid. Our analysis maintains higher order models for the inverters and the lines connecting them and is based on a representation of the grid dynamics in a common reference frame. By exploiting various decompositions of the network, input/output conditions have been derived that allow to exploit the coupling with neighbouring buses as well as the natural passivity properties of the lines. Appropriate homotopy arguments have been used to combine these conditions thus reducing the conservatism of the analysis. Examples of specific inverter control policies have also been discussed to demonstrate the results.

\section{APPENDIX}

\section{PROOF OF THEOREM 1}

Local asymptotic stability of the microgrid (3)-(4) equilibrium $\left.q\right|_{e q}$ is investigated via the internal stability [13, Def 5.2] of interconnection (5) [13, Thm 5.3], [14, Thm 3.7]. In particular, it is sufficient to show that the closed loop transfer functions of interconnection (5) (as defined in [13, Thm 5.3]) have no poles in the closed right half-plane.

The return ratio $R(\mathbf{j} \omega)$ of interconnection (5) is given by

$$
R(\mathbf{j} \omega)=\left(\mathcal{A} \otimes I_{2}\right)^{T} B(\mathbf{j} \omega)\left(\mathcal{A} \otimes I_{2}\right) L(\mathbf{j} \omega)
$$

and it has the same nonzero eigenvalues as

$$
\tilde{R}(\mathbf{j} \omega)=L(\mathbf{j} \omega)\left(\mathcal{A} \otimes I_{2}\right)^{T} B(\mathbf{j} \omega)\left(\mathcal{A} \otimes I_{2}\right) .
$$

Using ideas from [10], $\tilde{R}(\mathbf{j} \omega)$ can be rewritten as

$$
\tilde{R}(\mathbf{j} \omega)=\sum_{j=1}^{n_{n}} L(\mathbf{j} \omega) M_{j}\left(a_{j}^{r} \otimes I_{2}\right)^{T} B_{j}(\mathbf{j} \omega)\left(a_{j}^{r} \otimes I_{2}\right) M_{j}^{*}
$$

where

$$
M_{j}=\oplus_{k=1}^{n_{\ell}}\left(r_{k}^{j} I_{2}\right) \quad r_{k}^{j}= \begin{cases}1 & \text { if } \mathcal{A}_{j k} \neq 0 \\ 0 & \text { otherwise }\end{cases}
$$

Therefore, interconnection (5) can be represented equivalently as an interconnection of two systems $G(s)=$ $\oplus_{j=1}^{n_{b}} G_{j}(s)$ and $A$ such that

$$
\begin{aligned}
& y(s)=G(s) x(s) \\
& x(s)=-A y(s)
\end{aligned}
$$

with $G_{j}(s)$ given in Statement 1 and $A$ given by

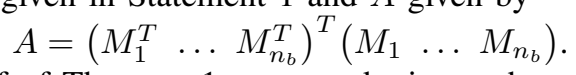

The proof of Theorem 1 uses quadratic graph separation arguments, analogous to an IQC analysis, on representations (5) and (11), together with a homotopy argument, with stability deduced using the multivariable Nyquist criterion.

The proof consists of three parts. First, we show that if Statement 1 is true for all $\omega \in \overline{\mathbb{R}}^{+}$, then interconnection (11) is stable. Then and in the same way, if Statement 2 is true for all $\omega \in \overline{\mathbb{R}}^{+}$then interconnection (5) is stable. Finally, we deduce that if at each frequency either of these statements is true then interconnection (5) is stable.

Part 1: Consider the interconnection (11). This first part is performed in three steps.

- Step 1: First, we show that if $-\Pi_{12}^{k}(\mathbf{j} \omega)-\Pi_{12}^{k}(\mathbf{j} \omega)^{*}+$ $2 \Pi_{22}^{k}(\mathbf{j} \omega) \leq 0$ and $\Pi_{11}^{k}(\mathbf{j} \omega) \leq 0$ then

$$
A \in \overline{\mathbf{Q C}}\left(I_{n_{b}} \otimes \Pi(\mathbf{j} \omega), 0\right) .
$$

Exploiting the sparsity structure of $A$, the left hand side of the expanded form of this condition is given by

$$
\begin{array}{r}
I_{n_{b}} \otimes\left(\oplus_{k=1}^{n_{l}} \Pi_{11}^{k}(\mathbf{j} \omega)\right)-M\left(\oplus_{k=1}^{n_{l}} \Pi_{12}^{k}(\mathbf{j} \omega)\right) M^{*}-\ldots \\
\ldots-M\left(\oplus_{k=1}^{n_{l}} \Pi_{12}^{k}(\mathbf{j} \omega)^{*}\right) M^{*}+M M^{*}\left(I_{n_{b}} \otimes \ldots\right. \\
\left.\cdots \otimes\left(\oplus_{k=1}^{n_{l}} \Pi_{22}^{k}(\mathbf{j} \omega)\right)\right) M M^{*}
\end{array}
$$

which is equal to

$$
\begin{gathered}
I_{n_{b}} \otimes\left(\oplus_{k=1}^{n_{l}} \Pi_{11}^{k}(\mathbf{j} \omega)\right)+M\left(\oplus _ { k = 1 } ^ { n _ { l } } \left(-\Pi_{12}^{k}(\mathbf{j} \omega)-\ldots\right.\right. \\
\left.\left.\ldots-\Pi_{12}^{k}(\mathbf{j} \omega)^{*}+\Pi_{22}^{k}(\mathbf{j} \omega) \sum_{j=1}^{n_{b}} r_{k}^{j}\right)\right) M^{*}
\end{gathered}
$$

Hence $-\Pi_{12}^{k}(\mathbf{j} \omega)-\Pi_{12}^{k}(\mathbf{j} \omega)^{*}+\Pi_{22}^{k}(\mathbf{j} \omega) \sum_{j=1}^{n_{b}} r_{k}^{j} \leq 0$ together with $\Pi_{11}^{k}(\mathbf{j} \omega) \leq 0$ are sufficient for (13) to be negative semi-definite and therefore for condition (12) to hold. Note that $r_{k}^{j}=1$ signifies that the $k^{t h}$ line is connected to the $j^{\text {th }}$ bus meaning that $\sum_{j=1}^{n_{b}} r_{k}^{j}$ is the number of buses connected to the $k^{t h}$ line. Therefore $\sum_{j=1}^{n_{b}} r_{k}^{j}=2$ for all $k \in \mathcal{E}$. 
- Step 2: We prove now that if condition (6) and condition (12) are satisfied, then the point -1 is not included in the eigenloci of $A G(\mathbf{j} \omega)$. Condition (6) can be written as

$$
G(\mathbf{j} \omega) \in \mathbf{Q C}\left(I_{n_{b}} \otimes \Pi(\mathbf{j} \omega), \epsilon_{G}(\omega)\right)
$$

for some $\epsilon_{G}(\omega)>0$ (the smallest $\epsilon_{G_{j}}(\omega)$ among all $j$ ).

Suppose that the point -1 is included in the eigenloci of $A G(\mathbf{j} \omega)$, that is $-1 \in \lambda_{i}(A G(\mathbf{j} \omega))$ and therefore $I_{2 n_{b} n_{\ell}}+A G(\mathbf{j} \omega)=0$. Then, there exists $x(\mathbf{j} \omega) \in \mathbb{C}^{2 n_{b} n_{\ell}}$ such that $\left(I_{2 n_{b} n_{\ell}}+A G(\mathbf{j} \omega)\right) x(\mathbf{j} \omega)=0$. Let $y(\mathbf{j} \omega)=G(\mathbf{j} \omega) x(\mathbf{j} \omega)$, the previous equality becomes $x(\mathbf{j} \omega)+A y(\mathbf{j} \omega)=0$ and the vector $\left(y(\mathbf{j} \omega)^{*} x(\mathbf{j} \omega)^{*}\right)^{*}$ can be given by $\left(G(\mathbf{j} \omega)^{*} \quad I_{2 n_{b} n_{\ell}}\right)^{*} x(\mathbf{j} \omega)$. As $x(\mathbf{j} \omega)=-A y(\mathbf{j} \omega)$ the same vector $\left(y(\mathbf{j} \omega)^{*} x(\mathbf{j} \omega)^{*}\right)^{*}$ is also given by $\left(I_{2 n_{b} n_{\ell}}-A^{*}\right)^{*} y(\mathbf{j} \omega)$. Pre and post multiplying the expanded forms of conditions (14) and (12) by $x(\mathbf{j} \omega)$ and $y(\mathbf{j} \omega)$ respectively results in

$\left(y(\mathbf{j} \omega)^{*} x(\mathbf{j} \omega)^{*}\right)\left(I_{n_{b}} \otimes \Pi(\mathbf{j} \omega)\right)\left(y(\mathbf{j} \omega)^{*} x(\mathbf{j} \omega)^{*}\right)^{*} \geq \epsilon_{G}(\omega) I_{2 n_{b} n_{\ell}}$ and

$$
\left(y(\mathbf{j} \omega)^{*} x(\mathbf{j} \omega)^{*}\right)\left(I_{n_{b}} \otimes \Pi(\mathbf{j} \omega)\right)\left(y(\mathbf{j} \omega)^{*} x(\mathbf{j} \omega)^{*}\right)^{*} \leq 0 ;
$$

which is a contradiction. Therefore, if conditions (6) and (12) are satisfied, then the point -1 is not included in the eigenloci of $A G(\mathbf{j} \omega)$.

- Step 3: Using the homotopy $A_{\tau}(\mathbf{j} \omega)=A$ and $G_{j, \tau}(\mathbf{j} \omega)=$ $\tau G_{j}(\mathbf{j} \omega)$ with $\tau \in[0,1]$, we show that condition (6) and condition (12) remains satisfied when replacing $G_{j}(\mathbf{j} \omega)$ by $G_{j, \tau}(\mathbf{j} \omega)$ and $A$ by $A_{\tau}$.

Constraint (12) is trivially satisfied in this homotopy. Condition (6) rewrites in its expanded form as

$$
W_{j}\left(\tau, G_{j}(\mathbf{j} \omega), \Pi(\mathbf{j} \omega)\right) \geq \epsilon_{G_{j}}(\omega) I_{2 n_{b}}
$$

with $W_{j}\left(\tau, G_{j}(\mathbf{j} \omega), \Pi(\mathbf{j} \omega)\right)=\tau^{2} G_{j}(\mathbf{j} \omega)^{*} \Pi_{11}(\mathbf{j} \omega) G_{j}(\mathbf{j} \omega)+$ $\tau\left(\Pi_{12}(\mathbf{j} \omega)^{*} G_{j}(\mathbf{j} \omega)+G_{j}(\mathbf{j} \omega)^{*} \Pi_{12}(\mathbf{j} \omega)\right)+\Pi_{22}(\mathbf{j} \omega)$.

This inequality is satisfied for $\tau=1$ from Step 2. It is also satisfied for $\tau=0$ if $\Pi_{22}(\mathbf{j} \omega) \geq \epsilon_{G_{j}}(\omega) I_{2 n_{b}}$. Furthermore, this inequality is satisfied for any $\tau \in[0,1]$ as $W_{j}\left(\tau, G_{j}(\mathbf{j} \omega), \Pi(\mathbf{j} \omega)\right)$ is concave in $\tau$ since $\Pi_{11}(\mathbf{j} \omega) \leq 0$. Therefore, conditions (6), (12) are satisfied when $G(\mathbf{j} \omega)$ and $A$ are replaced by $G_{\tau}(\mathbf{j} \omega)$ and $A_{\tau}$ respectively and hence the point -1 remains not included in the eigenloci of $A G(\mathbf{j} \omega)$. Therefore, starting from a stable interconnection corresponding to $\tau=0$ and continuously perturbing it until reaching $\tau=1$, ensures that the winding number of the point -1 does not change and hence there is no transition from stability to instability.

Therefore from the multivariable Nyquist criterion [15], it can be deduced that the closed loop transfer functions of interconnection (11) have no poles in the closed right halfplane.

Part 2: Consider the interconnection (5). From (3), it can be deduced that the line dynamics are passive which can be expressed in the frequency domain as $L_{k}(\mathbf{j} \omega) \in \overline{\mathbf{Q C}}\left(\Pi^{P}, 0\right)$, which implies that $L(\mathbf{j} \omega) \in$ $\overline{\mathbf{Q C}}\left(I_{n_{b}} \otimes \Pi^{P}, 0\right)$. Similarly, condition (7) is equivalent to $\left(\mathcal{A} \otimes I_{2}\right)^{T} B(\mathbf{j} \omega)\left(\mathcal{A} \otimes I_{2}\right) \in \mathbf{Q C}\left(I_{n_{b}} \otimes \Pi^{P}, \epsilon_{B}(\omega)\right)$ for some $\epsilon_{B}(\omega)>0$ (the smallest $\epsilon_{B_{j}}(\omega)$ among all $j$ ).
Using the same arguments as in Step 2 of Part 1, it follows that if the two previous conditions are satisfied then the point -1 is not encircled by the eigenloci of $\left(\mathcal{A} \otimes I_{2}\right)^{T} B(\mathbf{j} \omega)\left(\mathcal{A} \otimes I_{2}\right) L(\mathbf{j} \omega)$ ensuring that the closed loop transfer functions of interconnection (5) have no poles in the closed right half-plane.

Part 3: We deduce that if either of the two Statements in Theorem 1 is true at each frequency then both interconnections (5) and (11) have closed loop transfer functions with no poles in the closed right half-plane. This follows from the fact that the return ratio $A G(\mathrm{j} \omega)$ in interconnection (11) has the same nonzero eigenvalues as the return ratio $\left(\mathcal{A} \otimes I_{2}\right)^{T} B(\mathbf{j} \omega)\left(\mathcal{A} \otimes I_{2}\right) L(\mathbf{j} \omega)$ in interconnection (5). Hence the winding number of the point -1 will not change in both configurations when the $B_{j}(s)$ are multiplied by a scalar $\tau$ with $\tau \in[0,1]$, if either of the conditions in Statement 1, Statement 2 are satisfied at each frequency. Therefore the closed loop transfer functions of interconnection (5) have no poles in the closed right half-plane which leads to the proof of Theorem 1.

\section{REFERENCES}

[1] N. Hatziargyriou, H. Asano, R. Iravani, and C. Marnay, "Microgrids," IEEE power and energy magazine, vol. 5, no. 4, pp. 78-94, 2007.

[2] M. Farrokhabadi, C. A. Cañizares, J. W. Simpson-Porco, E. Nasr, L. Fan, P. A. Mendoza-Araya, R. Tonkoski, U. Tamrakar, N. Hatziargyriou, D. Lagos, et al., "Microgrid stability definitions, analysis, and examples," IEEE Transactions on Power Systems, vol. 35, no. 1, pp. 13-29, 2019.

[3] F. Milano, F. Dörfler, G. Hug, D. J. Hill, and G. Verbič, "Foundations and challenges of low-inertia systems," in 2018 Power Systems Computation Conference (PSCC), pp. 1-25, June 2018.

[4] V. Mariani, F. Vasca, J. C. Vásquez, and J. M. Guerrero, "Model order reductions for stability analysis of islanded microgrids with droop control," IEEE Transactions on Industrial Electronics, vol. 62, no. 7, pp. 4344-4354, 2014.

[5] J. Schiffer, D. Zonetti, R. Ortega, A. M. Stanković, T. Sezi, and J. Raisch, "A survey on modeling of microgrids - from fundamental physics to phasors and voltage sources," Automatica, vol. 74, pp. 135150, 2016.

[6] J. Schiffer, R. Ortega, A. Astolfi, J. Raisch, and T. Sezi, "Conditions for stability of droop-controlled inverter-based microgrids," Automatica, vol. 50, no. 10, pp. 2457-2469, 2014.

[7] Y. Ojo, J. Watson, and I. Lestas, "A review of reduced-order models for microgrids: Simplifications vs accuracy," arXiv preprint arXiv:2003.04923, 2020.

[8] C. Spanias and I. Lestas, "A system reference frame approach for stability analysis and control of power grids," IEEE Transactions on Power Systems, vol. 34, pp. 1105-1115, Mar 2019.

[9] J. Watson, Y. Ojo, C. Spanias, and I. Lestas, "Stability of power networks with grid-forming converters," in 2019 IEEE Milan PowerTech, pp. 1-6, June 2019.

[10] I. Lestas, "On network stability, graph separation, interconnection structure and convex shells," IEEE Conf. Decision and Control, pp. 4257-4263, 2011.

[11] T. Iwasaki and S. Hara, "Generalized kyp lemma: Unified frequency domain inequalities with design applications," IEEE Transactions on Automatic Control, vol. 50, no. 1, pp. 41-59, 2005

[12] Y. Sun, X. Hou, J. Yang, H. Han, M. Su, and J. M. Guerrero, "New perspectives on droop control in ac microgrid," IEEE Transactions on Industrial Electronics, vol. 64, no. 7, pp. 5741-5745, 2017.

[13] K. Zhou, J. Doyle, and K. Glover, Robust and Optimal Control. Prentice Hall, New Jersey, 1995.

[14] H. Khalil, Nonlinear Systems. New York: Macmillan, 1992.

[15] C. Desoer and Yung-Terng Wang, "On the generalized Nyquist stability criterion," IEEE Transactions on Automatic Control, vol. 25, pp. 187-196, Apr. 1980. 